\title{
Trifluoperazine an Antipsychotic Drug and Inhibitor of Mitochondrial Permeability Transition Protects Cytarabine and Ifosfamide-Induced Neurotoxicity
}

\author{
Authors \\ Amir Kiani ${ }^{1}$, Shadi Heydari Nik ${ }^{1}$, Adineh Khodadoost ${ }^{1}$, Ahmad Salimi ${ }^{2}$, Jalal Pourahmad ${ }^{3}$
}

Affiliations

1 Department of Pharmacology and Toxicology, School of Pharmacy, Kermanshah University of Medical Sciences, Kermanshah, Iran

2 Department of Pharmacology and Toxicology, School of Pharmacy, Ardabil University of Medical Sciences, Ardabil, Iran

3 Department of Pharmacology and Toxicology, Faculty of Pharmacy, Shahid Beheshti University of Medical Sciences, Tehran, Iran

\section{Key words}

trifluoperazine, cytarabine, ifosfamide, neurotoxicity, mitochondria

$\begin{array}{ll}\text { received } & 24.02 .2020 \\ \text { revised } & 18.03 .2020 \\ \text { accepted } & 23.03 .2020\end{array}$

\section{Bibliography}

DOI https://doi.org/10.1055/a-1154-8672

Published online: 4.5 .2020

Drug Res 2020; 70: 265-272

(c) Georg Thieme Verlag KG Stuttgart · New York

ISSN 2194-9379

\section{Correspondence}

Jalal Pourahmad

Prof. of Toxicology and Pharmacology, Faculty of Pharmacy,

Shahid Beheshti University of Medical Sciences,

14155-6153 Tehran, Iran

Tel.: + 98(21)2255-8786, Fax: + 98(21)8820-9620

j.pourahmadjaktaji@utoronto.ca
Ahmad Salimi

Assistant Professor of Toxicology and Pharmacology, School of Pharmacy, Ardabil University of Medical Sciences, 56189-53141 Ardabil, Iran

Tel.:+98(45)3352-3833, Fax: + 98(45)3352-2197

salimikd@yahoo.com; a.salimi@pharmacy.arums.ac.ir

\section{ABSTRACT}

The link between $\mathrm{Ca}^{2+}$ dysregulation, mitochondria damages, oxidative stress and cellular derangement is particularly evident in neurotoxicity induced by chemotherapeutic agents. In the current study, we investigated effects of trifluoperazine (TFP) as an inhibitor of calmodulin against the cytotoxicity induced by cytarabine (Ara-C) and Ifosfamide (IFOS) on isolated rat neurons and also the mechanisms involved in this toxicity. Isolated rat neurons were pretreated with TFP $(100 \mu \mathrm{M})$ for $5 \mathrm{~min}$ at $37^{\circ} \mathrm{C}$, then Ara-C $(226 \mu \mathrm{M})$ and IFOS $(290 \mu \mathrm{M})$ were added in separate experiments. After $3 \mathrm{~h}$, the cytotoxicity, reactive oxygen species (ROS), lysosomal membrane destabilization, mitochondrial membrane potential (MMP), lipid peroxidation (LP), glutathione (GSH) and glutathione disulfide (GSSG) levels were measured. Ara-C and IFOS treatments caused a significant decrease in cellular viability, which was accompanied by ROS generation, GSSG/GSH ratio, lipid peroxidation and lysosomal and mitochondrial damages. On the other hand, TFP $(100 \mu \mathrm{M})$ pre-treatment attenuated Ara-C and IFOS -induced decrease in cell viability. In addition, TFP $(100 \mu \mathrm{M})$ pre-treatment significantly protected against Ara-C and IFOS -induced increase in ROS generation, lysosomal and mitochondrial damages, lipid peroxidation levels and decrease in GSH/GSSG ratio. Our data provided insights into the mechanism of protection by TFP against Ara-C and IFOS neurotoxicity, which is related, to neuronal ROS formation and mitochondrial damages.

\section{Introduction}

Chemotherapy induced neurotoxicity is the main limitations in cancer patients [1]. Around $30-40 \%$ of patients undergoing chemotherapy experience neurotoxicity, sensory disturbances and symptoms of pain [2]. The most frequent agents causing neurotoxicity are chemotherapy agents [2]. A common mechanism underlying the neurotoxicity is physical damage to the neurons by chemother- apeutic agent [3]. The physical damage induced by anticancer drugs leads to mitochondrial damages, oxidative stress, inflammation, apoptosis, electrophysiological disturbances functional and impairment in neurons [3]. Chemotherapeutic agents produce ROS and induce apoptosis in cancer cells [4]. However, ROS produced during cancer therapy may intervene with the normal tissues and cells and may lead to the various toxic events like neurotoxicity, ne- 
phrotoxicity cardio toxicity and other toxicities. Mammalian nerves are well-known to be more sensitive to oxidative stress due to their mitochondria rich axoplasm weak cellular antioxidant defenses and high content of phospholipids [5]. It has been reported that functional and structural deteriorations caused by chemotherapeutic agents enhance mitochondrial free radical production [6]. Oxidative stress caused by mitochondria pathway lead to bioenergetic failure, depletion of antioxidant defenses, mitochondrial dysfunction, bio molecular damage, microtubular disruption, neuroinflammation, mitophagy impairment, ion channel activation, demyelination, and finally neuronal death through apoptosis [7]. Accumulation of dysfunctional mitochondria due to chemotherapeutic agents increase the vicious cycle of oxidative damage to the mitochondria and bio molecules that leads a feed-forward mechanism and more accumulation of ROS and reactive nitrogen species (RNS) in the neurons [8]. In this study, we focused on two main chemotherapeutic drugs, cytarabine and ifosfamid with neurotoxicity potential.

Ara-C, as first line chemotherapy is used in treatment of hematological cancers especially in acute myeloid leukemia [9]. A recent study showed that cytarabine inhibits DNA polymerase $y$ and leads to reduction in mitochondrial DNA (mtDNA) content, ROS generation and oxidative damage in neurons. This study suggests that cytarabine neurotoxicity in neurons originates in mitochondria and continuous with oxidative stress [10]. Also, IFOS, a structural analog of cyclophosphamide, is an alkylating chemotherapy agent used for a wide range of solid and hematologic malignancies [10]. IFOS has been reported to have adverse neurological effects [11]. Limited works were done to address mechanisms underlying neurotoxicity of IFOS. It has been suggested that chloroethylamine as an IFOS metabolite induce the formation of thialysine ketamine which inhibits electron-binding flavoproteins in the mitochondrial respiratory chain that probably leads to mitochondrial damages and oxidative stress [12]. Oxidative stress and mitochondrial damages have been reported of cyclophosphamide as a structural analog IFOS [13].

TFP, a long-established high potency typical antipsychotic drug used in the treatment of schizophrenia-like and schizophrenia illnesses. Also, TFP as an inhibitor of calmodulin, can reduce the celIular and mitochondrial $\mathrm{Ca}^{2+}$ overload $[14,15]$. It has been reported that TFP can interact with the inner membrane of mitochondria, acquired antioxidant activity toward processes with potential toxicity in cell death, such as ROS formation, lipid peroxidation of the membrane and MMP collapse and release of cytochrome c [16]. Several studies have also been reported that TFP, significantly protected mitochondria against the deleterious effects of $\mathrm{Ca}^{2+}$ and hydrogen peroxide $[17,18]$. The aim of our study was therefore to explore the effects of Ara-C and IFOS on isolated rat neurons as well as assessing the protective effects of TFP against Ara-C and IFOS -induced oxidative stress and mitochondrial damage.

\section{Materials and Methods}

\section{Animals}

Clean grade wistar rats weighting $150-200 \mathrm{~g}$ were purchased from the Pasteur Institute of Iran (Tehran, Iran). This study was approved by the Research Ethics Committee at the Shahid Beheshti University of Medical Sciences, and performed strictly in accordance with institutional and international guide for animal care.

\section{Chemicals}

Fetal Bovine Serum (FBS), B-27TM supplement (50X), Neurobasal TM medium, serum free medium, Dulbecco's Modified Eagle Medium (DMEM), Trypsin, 3-(4,5-dimethylthiazol-2-yl)-2,5-diphenyltetrazolium bromide (MTT), Ethylenediaminetetraacetic Acid (EDTA), $\beta$-NGF and L-glutamine $(200 \mathrm{mM})$ were purchased from GIBICO (Gaithersburg, MD, USA). Trifluoperazine (TFP), cytarabine (Ara-C) and Ifosfamide (IFOS) was purchased from Abidi Pharmaceutical Co. (Tehran, Iran). L- carnitine (LC), Sodium Pyruvate, Glutamine, Hank's Balanced Salt Solution (HBSS), trypan blue, 2', 7' Dichlorofuorescin Diacetate (DCFH-DA), Acridine Orange (AO), N-ethylmaleimide (NEM), Rhodamine123, N-(2-hydroxyethyl) piperazine- $\mathrm{N}^{\prime}$-(2-ethanesulfonic acid) (HEPES), bovine serum albumin (BSA), were obtained from the Chemical Co. were purchased from Sigma (Cambridge, MA, USA).

\section{Isolation of primary rat brain neurons}

The rats were sacrificed by cervical dislocation. The brain was removed and put in HBSS buffer. The blood vessels and pia mater were removed. The brain was incubated in $0.125 \%$ trypsin at $37^{\circ} \mathrm{C}$ for 10 - 15 min, rinsed twice with HBSS, and prepared into cell suspension in DMEM containing $10 \%$ FBS. The cell suspension was filtered through a sterile $70 \mu \mathrm{m}$ filter, and seeded into DMEM supplemented with $10 \% \mathrm{FBS}, 1 \%$ sodium pyruvate and $1 \%$ glutamine. After $4 \mathrm{~h}$, the medium was replaced with neurobasal medium containing $2 \%$ B27 and $1 \%$ glutamine [19].

\section{Cell viability assay}

The cell viability was measured by the MTT assay. Cells at $70 \%$ confluence in 6-well plates were incubated for $3 \mathrm{~h}$ in normal medium or medium with different concentration of Ara-C (0-1000) and IFOS (0-1000) or IC50 $3 \mathrm{~h}$ Ara-C/IFOS + $100 \mu \mathrm{M}$ TFP. Cells were collected and stained using trypan blue exclusion dye under optical microscope at 10x.

\section{Measurement of ROS}

The rate of ROS generation was evaluated by using the probe DCFHDA. In the presence of ROS, DCFH is oxidized to highly fluorescent dichlorofluorescein (DCF). Cells at $70 \%$ confluence were treated for 1,2 and $3 \mathrm{~h}$ in normal medium or medium with different concentrations of Ara-C (113, 226 and $452 \mu \mathrm{M})$ or IFOS (145, 290 and $580 \mu \mathrm{M})$ or IC50 $3 \mathrm{~h}$ Ara-C/IFOS $100 \mu \mathrm{M}$ TFP. After the incubation time, medium was replaced by $10 \mu \mathrm{M}$ DCFH-DA containing medium, after $15 \mathrm{~min}$ incubation, the fluorescence intensity was measured by fluorescence spectrophotometer (Shimadzu RF5000U) at the excitation wavelength of $495 \mathrm{~nm}$ and the emission wavelength of $530 \mathrm{~nm}[20]$.

\section{Measurement of MMP collapse}

The change in the MMP in the isolated neurons were measured by using the cationic fluorescent dyerhodamine- 123 . Cells at $70 \%$ confluence were treated for 1,2 and $3 \mathrm{~h}$ in normal medium or medium 
with different concentrations of Ara-C $(113,226$ and $452 \mu \mathrm{M})$ or IFOS $(145,290$ and $580 \mu \mathrm{M})$ or IC50 $3 \mathrm{~h}$ Ara-C/IFOS + $100 \mu \mathrm{M}$ TFP. After the incubation time, medium was replaced by $1 \mu \mathrm{M}$ rhodamine- 123 containing medium, after 15 min incubation, the medium was removed, and the fluorescence intensity was measured by fluorescence spectrophotometer (Shimadzu RF5000U) at the excitation wavelength of $470 \mathrm{~nm}$ and the emission wavelength of $540 \mathrm{~nm}[21]$.

\section{Measurement of lysosomal membrane destabilization}

The isolated neurons lysosomal membrane integrity was assessed from the redistribution of the lipophilic dye acridine orange. Cells at $70 \%$ confluence were treated for 1,2 and $3 \mathrm{~h}$ in normal medium or medium with different concentrations of Ara-C (113, 226 and $452 \mu \mathrm{M})$ or IFOS $(145,290$ and $580 \mu \mathrm{M})$ or IC50 $3 \mathrm{~h}$ Ara-C/IFOS + $100 \mu \mathrm{M}$ TFP. After the incubation time, medium was replaced by $5 \mu \mathrm{M}$ acridine orange containing medium. After 10 min incubation, the fluorescence intensity was measured by fluorescence spectrophotometer (Shimadzu RF5000U) at the excitation wavelength of $470 \mathrm{~nm}$ and the emission wavelength of $540 \mathrm{~nm}$ [22].

\section{Measurement of lipid peroxidation}

Lipid peroxidation was measured by using the thiobarbituric acid assay and malondialdehyde (MDA) formation. The cells were exposed for 1,2 and $3 \mathrm{~h}$ with different concentrations of Ara-C (113, 226 and $452 \mu \mathrm{M})$ or IFOS $(145,290$ and $580 \mu \mathrm{M})$ or IC50 $3 \mathrm{~h} \mathrm{Ara-C/}$ IFOS + $100 \mu \mathrm{M}$ TFP. After the incubation, cells were washed with PBS, and then lysed with PBS contain $2 \%$ triton. $100 \mu$ l of cell lysate was mixed with $200 \mu$ of thiobarbituric acid (TBA) reagent (containing $3.75 \%$ TCA and $0.0925 \%$ TBA) and the mixture was incubated at $90^{\circ} \mathrm{C}$ for $60 \mathrm{~min}$. After cooling, the mixture was centrifuged at $1000 \times \mathrm{g}$ for $10 \mathrm{~min}$. Calorimetric absorption was measured at $530 \mathrm{~nm}[23]$.

\section{Measurement of GSH and GSSG}

GSH and GSSG levels in Ara-C/IFOS-treated isolated neurons were measured by Hissin and Hilf method [24]. After treatment of isolated neurons with different concentrations of Ara-C $(113,226$ and $452 \mu \mathrm{M})$ or IFOS $(145,290$ and $580 \mu \mathrm{M})$ or IC50 $3 \mathrm{~h}$ Ara-C/IFOS + $100 \mu \mathrm{M}$ TFP, the cells were lysed with $0.5 \mathrm{ml}$ of trichloroacetic acid (TCA) $10 \%$ and centrifuged at $11000 \times \mathrm{g}$ for $2 \mathrm{~min}$. For assessment of GSH, supernatant was diluted with phosphate-EDTA buffer and incubated with $100 \mu$ of the o-phthalaldehyde (OPT) solution for 15 min at room temperature. For determination of GSSG, cells supernatant was diluted with $\mathrm{NaOH} 0.1 \mathrm{~N}$ solution and before incubation with OPT, $200 \mu$ l of $\mathrm{N}$-ethylmaleimide (NEM) solution was incubated with supernatant for $30 \mathrm{~min}$. The fluorescence intensity was measured by fluorescence spectrophotometer (Shimadzu RF5000U) at the excitation wavelength of $350 \mathrm{~nm}$ and the emission wavelength of $420 \mathrm{~nm}$.

\section{Statistical analysis}

All data are presented as the Mean \pm Standard Deviation (SD) with three separate experiments. Data were analyzed by GraphPad Prism 5 (GraphPad Software, La Jolla, CA) using one and two-way analysis of variance followed by post hoc Tukey and Bonferroni test. P value of less than 0.05 was considered as statistically significant.

\section{Results}

\section{Cell Viability}

Cytotoxic effects of Ara-C (0-1000 $\mu \mathrm{M})$ or IFOS $(0-1000 \mu \mathrm{M})$ on isolated neurons showed in the $\mathbf{F i g} \mathbf{1} \mathbf{a} \mathbf{a} \mathbf{b}$. Ara-C and IFOS caused dose dependent cytotoxicity on the cells and significantly $(P<0.05)$ reduced cell viability in all used concentrations. The presented data at $>$ Fig. 1 c-d demonstrated that TFP $(100 \mu \mathrm{M})$, as a mitoprotective agents prevent of cytotoxicity induced by Ara-C and IFOS.

\section{ROS production}

The effects of Ara-C (113, 226 and $452 \mu \mathrm{M})$ or IFOS (145, 290 and $580 \mu \mathrm{M})$ on the generation of ROS in isolated neurons are shown in - Fig. 2 a-b. Ara-C/IFOS has induced dose and time dependent ROS generation in isolated neurons. When the isolated neurons were simultaneously treated with Ara-C/IFOS + TFP $(100 \mu \mathrm{M})$, the mean fluorescence intensities were significantly decreased compared to treated groups with Ara-C/IFOS ( $\triangleright$ Fig. 2 a-b).

\section{MMP collapse}

The effects of Ara-C (113, 226 and $452 \mu \mathrm{M})$ or IFOS (145, 290 and $580 \mu \mathrm{M})$ on the MMP of isolated rat neurons were presented in the Fig. 3 a-b. Ara-C/IFOS induced statistically $(\mathrm{P}<0.05)$ MMP collapse in dose and time dependent manner. As shown in the Fig. $\mathbf{3}$ a-b, collapse of mitochondrial membrane potential was inhibited after treatment of neurons with Ara-C/IFOS by TFP $(100 \mu \mathrm{M})$ at toxic doses.

\section{Lysosomal membrane destabilization}

The effect of Ara-C (113, 226 and $452 \mu \mathrm{M})$ or IFOS (145, 290 and $580 \mu \mathrm{M})$ showed in $>$ Fig. 4a-b. Ara-C/IFOS induced statistically lysosomal damages in dose and time dependent manner. Lysosomal membrane destabilization was inhibited after treatment of isolated neurons with Ara-C/IFOS by TFP $(100 \mu \mathrm{M})$ at toxic doses ( $\triangleright$ Fig. 4 a-b).

\section{Lipid peroxidation}

Lipid peroxidation as an indicator of oxidative damage to the lipids was measured in isolated neurons using MDA assay as a byproduct of lipid peroxidation. We showed that the amount of MDA as the result of lipid peroxidation significantly $(P<0.05)$ increased when cells incubated Ara-C $(113,226$ and $452 \mu \mathrm{M})$ or IFOS $(145,290$ and $580 \mu \mathrm{M})$ at toxic dose during $1-3 \mathrm{~h}$ ( $>$ Fig. 5 a-b). Pretreatment of isolated neurons with by TFP $(100 \mu \mathrm{M})$ at toxic doses significantly $(\mathrm{P}<0.05)$ decreased the MDA level after $3 \mathrm{~h}$. incubation time ( Fig. $\mathbf{5}$ a-b).

\section{GSH and GSSG content}

Isolated neurons were treated with Ara-C (113, 226 and $452 \mu \mathrm{M})$ or IFOS (145, 290 and $580 \mu \mathrm{M})$ and $1 \mathrm{~h}$ after treatment decrease in GSH/GSSG ratio were observed. The effects of Ara-C/IFOS on GSH and GSSG content are shown in $>$ Fig. $\mathbf{6}$ a-b. This finding indicates a significant $(P<0.001)$ changes in GSH/GSSG ratio in concentration-dependent manner. Pretreatment of isolated neurons with TFP $(100 \mu \mathrm{M})$ inhibited decrease of GSH/GSSG ratio. GSH/GSSG 

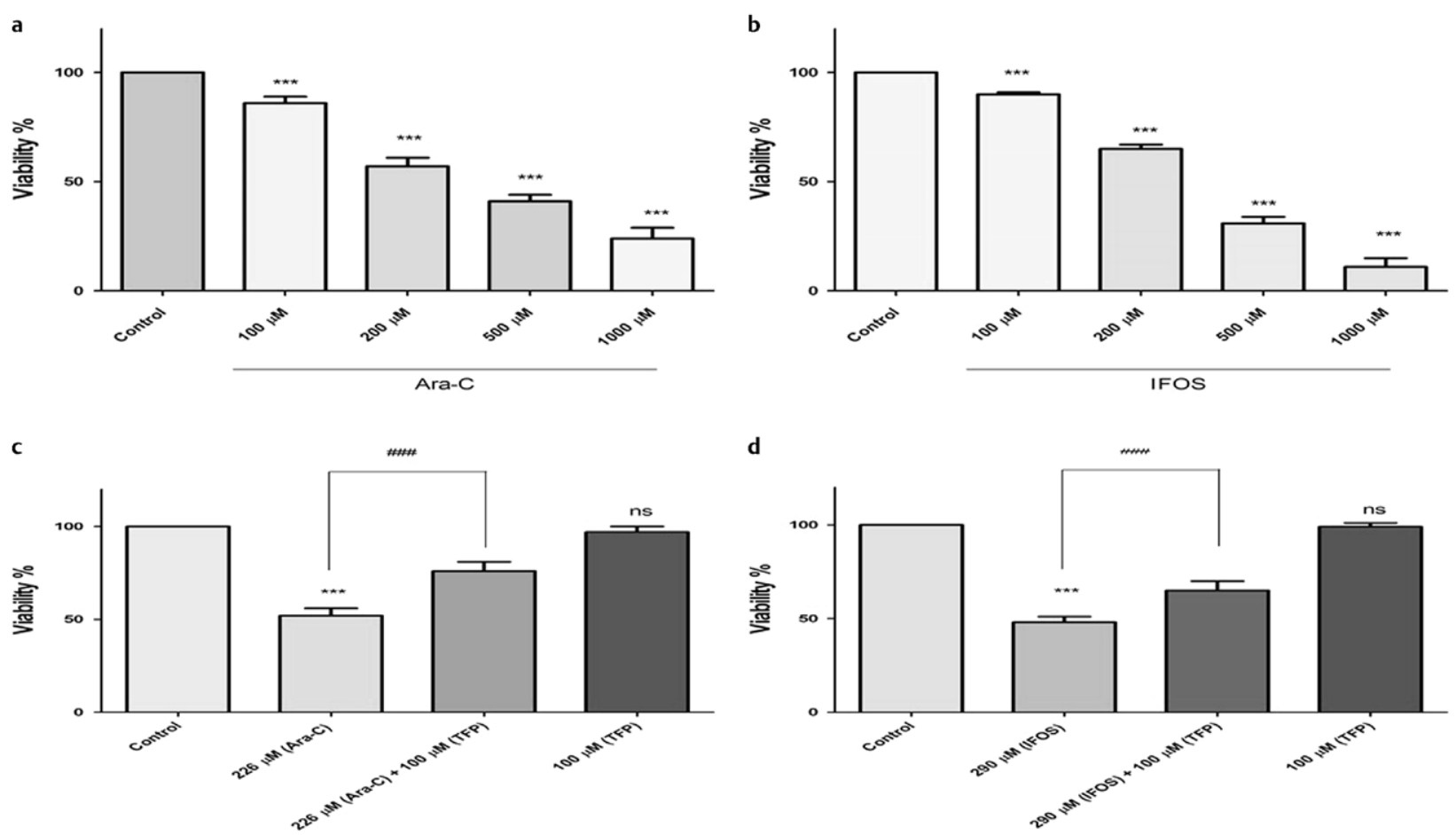

- Fig. 1 Viability of isolated neurons following treatment with Ara-C/IFOS for $3 \mathrm{~h}$ ( $\mathbf{a}$ and $\mathbf{b}$ ) and protective effect of TFP against Ara-C/IFOS toxicity (c and d). Cell viability determined by trypan blue assay after incubation of the cells with different concentration of Ara-C/IFOS. Ara-C and IFOS both decrease neuron viability in a dose-dependent manner and this decrease is significant at concentration higher than $100 \mu \mathrm{M}$. $\left({ }^{* * *} p<0.001 \mathrm{vs}\right.$. control) (a and $\mathbf{b})$. Protective effect of TFP $(100 \mu \mathrm{M})$ are tested against cytotoxicity induced Ara-C/IFOS (c and d). Presented data showed these agents significantly decreased cell death compared to treated groups with Ara-C/IFOS alone. ( ${ }^{* * *} p<0.001$ vs. control, \#\#\#p<0.001 vs. treated groups with Ara-C/IFOS).
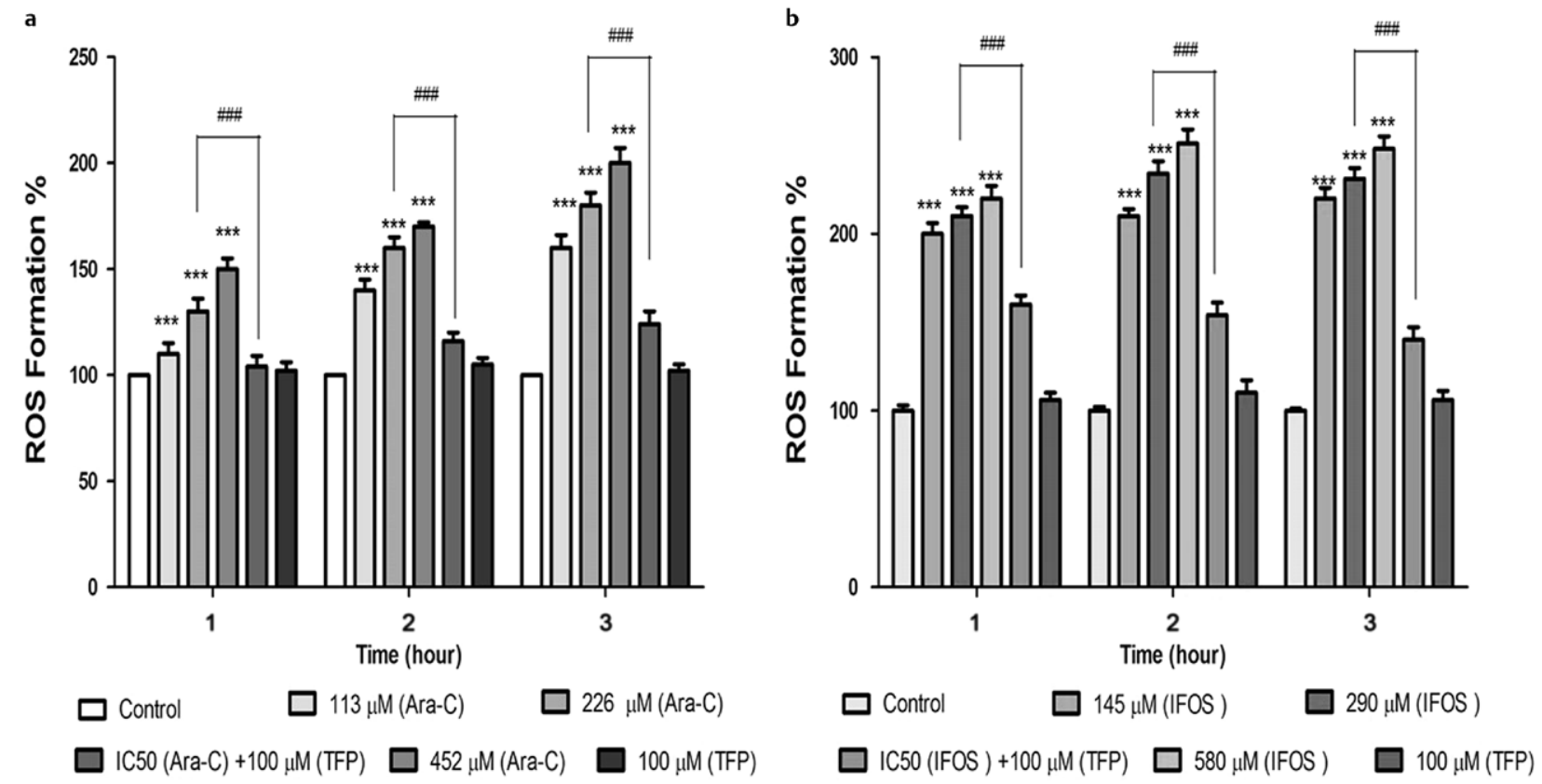

-Fig. 2 ROS Generation in isolated neurons after incubation with Ara-C/IFOS for different concentrations and time (1,2 and $3 \mathrm{~h}$ ) intervals and protective effect of TFP against Ara-C/IFOS induced ROS formation (a-b). Ara-C/IFOS has induced dose and time dependent ROS production (a-b). TFP, inhibited Ara-C/ IFOS -induced generation of ROS in isolated neurons. ( ${ }^{* * *} p<0.001$ vs. control, \#\#\#p<0.001 vs. treated groups with Ara-C/IFOS). 

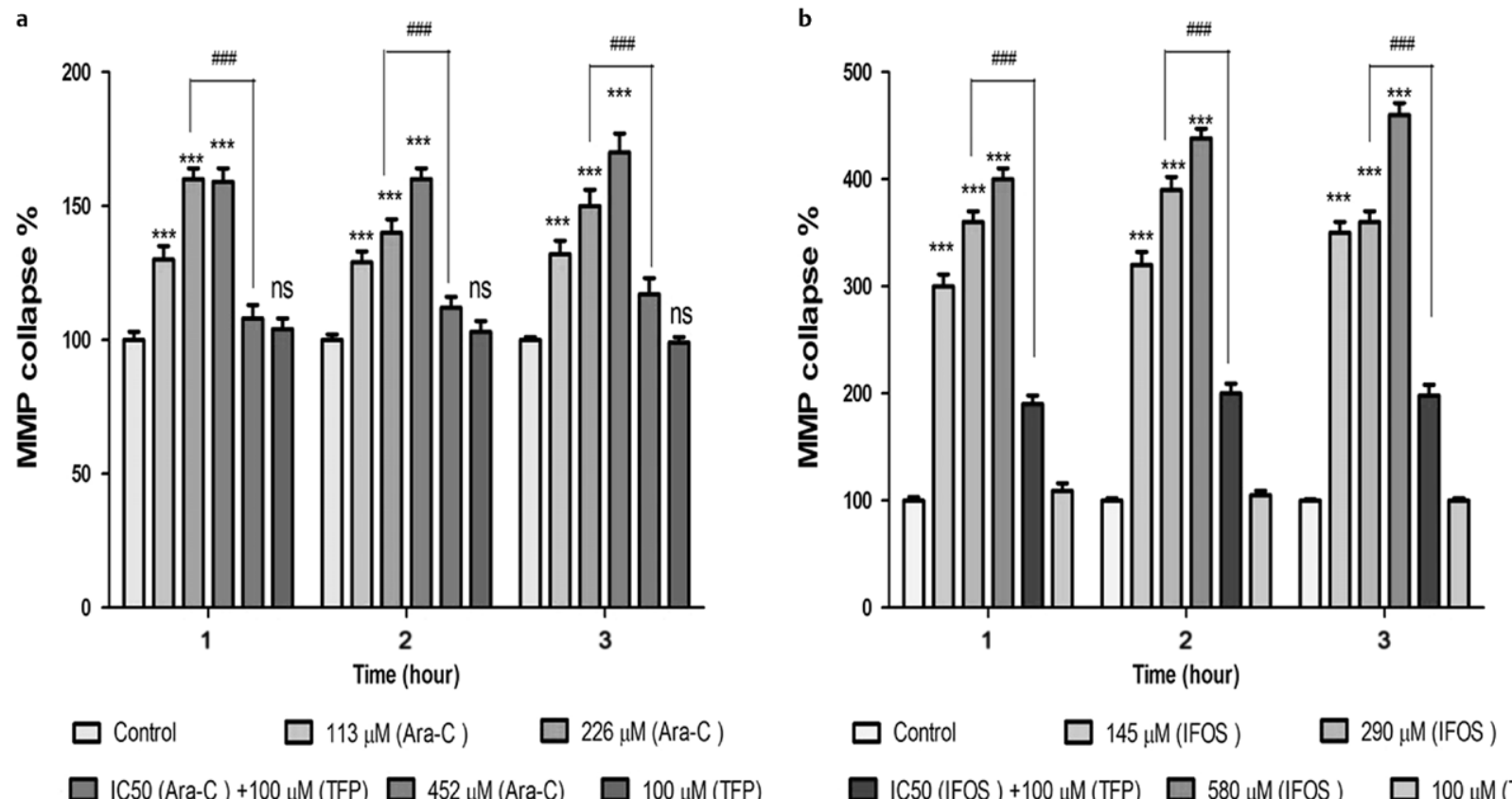

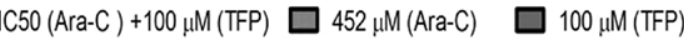

IC50 (IFOS) $+100 \mu \mathrm{M}$ (TFP)

$580 \mu \mathrm{M}(\mathrm{IFOS})$

$100 \mu \mathrm{M}($ TFP)

- Fig. 3 Collapse of mitochondrial membrane potential (MMP) in isolated neurons following incubation with Ara-C/IFOS for 1,2 and $3 \mathrm{~h}$ and protective effect of TFP against Ara-C/IFOS induced mitochondrial damages (a-b). Collapse in mitochondrial membrane potential started $1 \mathrm{~h}$ after treatment of isolated neurons with Ara-C/IFOS at three concentrations. Ara-C/IFOS-induced mitochondrial membrane potential collapse was time and

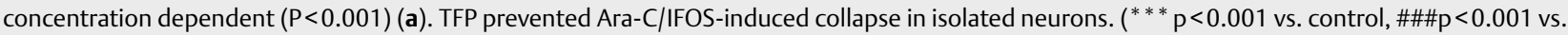
treated groups with Ara-C/IFOS).
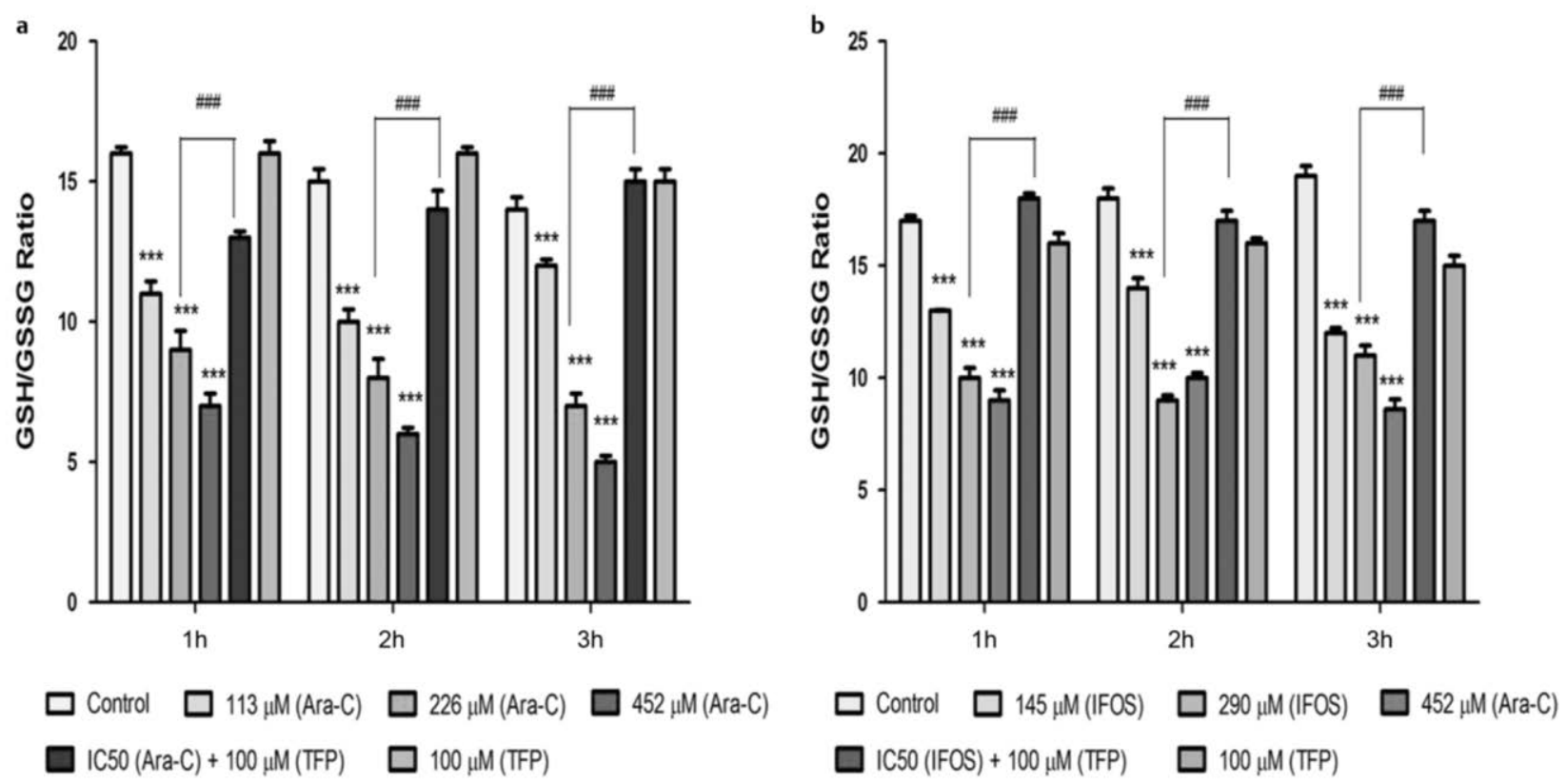

-Fig. 4 Lysosomal membrane destabilization in isolated neurons after incubation with Ara-C/IFOS and protective effect of TFP against Ara-C/ IFOS-induced lysosomal damages (a-b). After 1,2 and $3 \mathrm{~h}$ treatment, Ara-C/IFOS caused significant $(\mathrm{P}<0.001)$ lysosomal membrane leakage (a). TFP prevented Ara-C/IFOS-induced lysosomal membrane leakage. $\left({ }^{* * *} \mathrm{p}<0.001\right.$ vs. control, \#\#\#p<0.001 vs. treated groups with Ara-C/IFOS). 


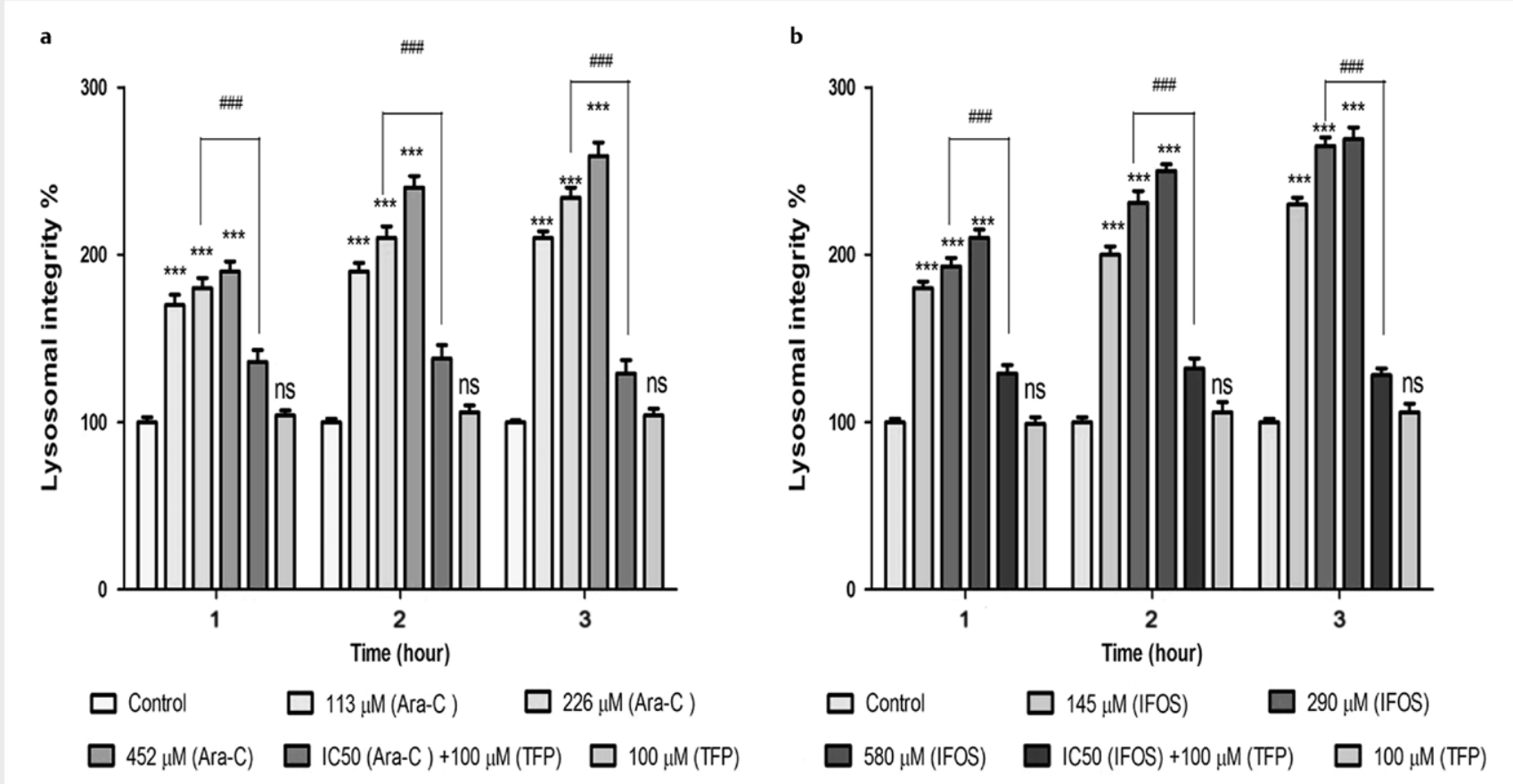

-Fig. 5 Induction of lipid peroxidation in isolated neurons and after incubation with Ara-C/IFOS and protective effect of TFP against Ara-C/IFOSinduced oxidative damages (a-b). Lipid peroxidation significantly increased when isolated neurons were incubated with Ara-C/IFOS (a). TFP prevented Ara-C/IFOS-induced lipid peroxidation. ( ${ }^{* * *} \mathrm{p}<0.001$ vs. control, \#\#\# $<0.001$ vs. treated groups with Ara-C/IFOS).
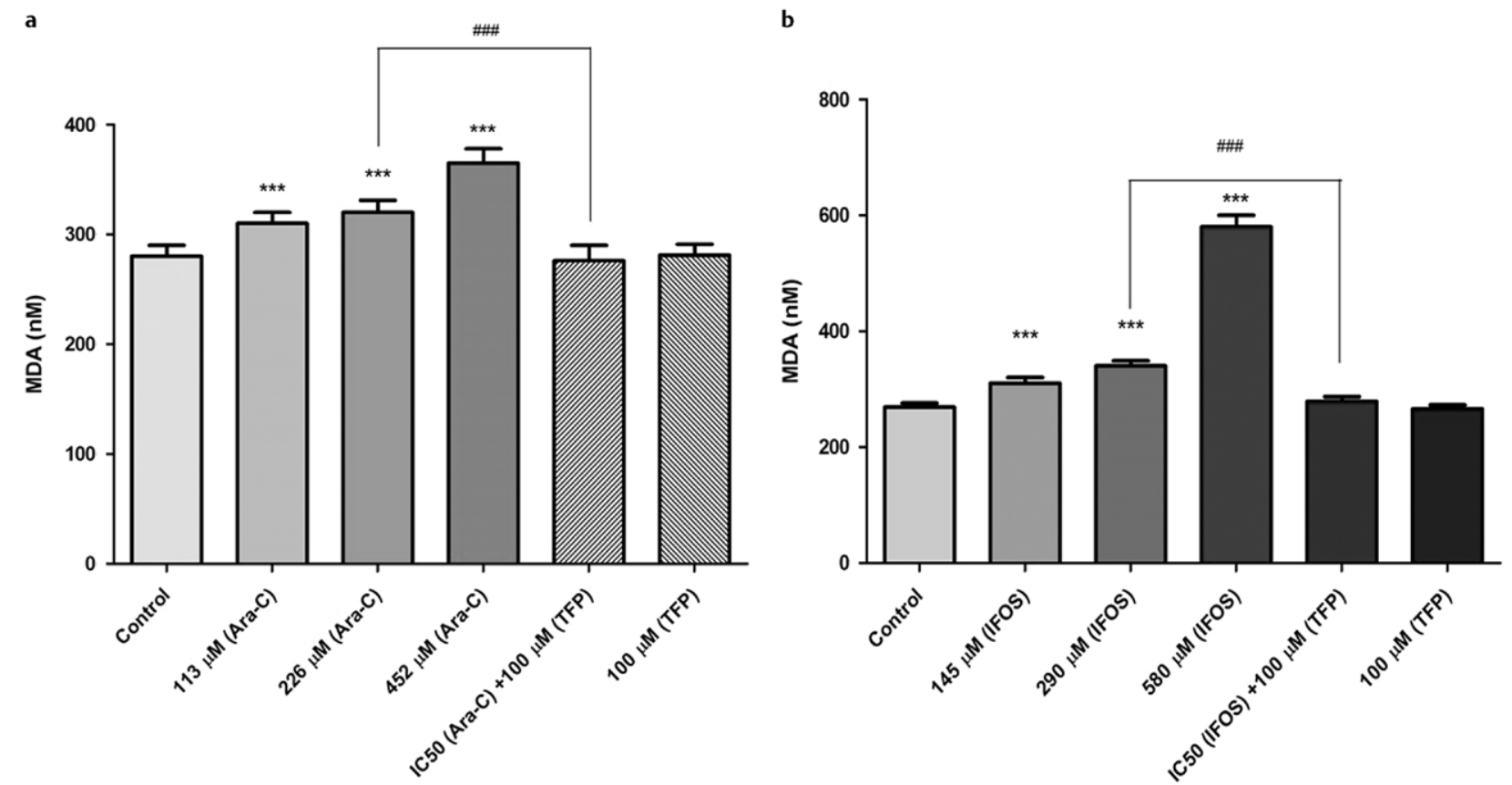

- Fig. 6 Effect of Ara-C/IFOS on GSH/GSSG ratio and protective effect of TFP against Ara-C/IFOS-induced GSH depletion (a-b). As demonstrated, significant $(\mathrm{P}<0.001) \mathrm{GSH} / \mathrm{GSSG}$ ratio decrease was found after treatment with Ara-C/IFOS. TFP prevented depletion of GSH $\left({ }^{* * *} \mathrm{P}<0.001\right.$ vs. control, \#\#\#p<0.001 vs. treated groups with Ara-C/IFOS). 
ratio significantly $(P<0.05)$ increased at 1,2 and $3 \mathrm{~h}$ following cotreatment with Ara-C/IFOS + TFP in isolated neurons.

\section{Discussion}

The reactive oxygen species are produced in the cell through several pathways. The generated ROS within cells are rapidly removed by various non-enzymatic and enzymatic mechanisms [25]. Disruption of the antioxidant-oxidant equivalence originates oxidative stress and cell damage [25]. The oxidative stress produced by anticancer drugs cause side effects in the treated patients [26]. When the formation of ROS oversteps repair capacities, cellular adaptive biological molecules such as proteins, membrane phospholipids and nucleic acids, become damaged because of oxidative reactions. Finally, oxidative stress leads to the defeat of normal cellular functions and even cell death [27]. Methotrexate, Ara-C, and IFOS are the anticancer drugs that most frequently cause central nervous system (CNS) toxicity. These agents induce central neurotoxic adverse effects [28]. In the current study, we focused on cytotoxicity of Ara-C, and IFOS on isolated brain neurons. Our measured toxicity parameters showed that Ara-C, and IFOS significantly induce ROS formation. Previous studies showed that Ara-C, and IFOS increase the ROS formation and oxidative stress in normal and tumor cells $[29,30]$. Also, other examinations showed that both drugs induce lipid peroxidation and decrease GSH in isolated neurons. Our results are consistent with previously published data examining Ara-C, and IFOS-induced oxidative stress in several systems [29, 30].

Drug-induced mitochondrial toxicity has been investigated well for over 50 years in academic settings. Drugs may inhibit mitochondrial function in many different ways [31]. Mitochondrial toxicity has been identified to cause organ toxicity to the central nervous system, kidney, skeletal muscle, heart and liver [32]. Drug classes identified to cause mitochondrial toxicity are anti-diabetic, cholesterol lowering, anti-depressants, pain medications, certain antibiotics, and anti-cancer drugs [33]. Most of drug-induced mitochondrial toxicities were not detected in preclinical animal studies. Most of these effects have been proven through studies in isolated cells and mitochondria [33]. Our results showed that Ara-C, and IFOS significantly induce mitochondrial membrane potential collapse in isolated rat neurons, which is consistent with previously published data examining anticancer drugs-induced mitochondrial toxicity in other tissues.

Lysosomes serve as the cellular recycling center and are filled with numerous hydrolases that can degrade most cellular macromolecules [34]. Lysosomal membrane permeabilization and the consequent leakage of the lysosomal content into the cytosol leads to lysosomal cell death [34]. This form of cell death is mainly carried out by the lysosomal cathepsin proteases and can have apoptotic, apoptosis-like or necrotic features depending on the extent of the leakage and the cellular context [34]. Many lipophilic, weakly basic drugs accumulate in lysosomes and apply complex, pleiotropic effects on organelle structure and function [35]. In the current study we observed lysosomal membrane permeabilization after exposure of isolated neurons with Ara-C, and IFOS. However, this effect may be due to the production of reactive oxygen species or mitochondrial damage caused by these drugs which is known as lysosomal and mitochondrial crosstalk [36].
There are conflicting opinions regarding the administration of antioxidants during cancer therapy [37]. Antioxidants may reduce the effectiveness of chemotherapies, which are based on increasing oxidative stress in tumor cells [37]. For example, Ara-C was toxic to $\mathrm{MLH} 1$ and $\mathrm{MLH} 2$ deficient tumor cells, but this cytotoxicity was reduced by antioxidants [30]. Also, certain researchers revealed mitochondrial dysfunction and mitotoxicity contribute to amplified oxidative stress [38]. Therefore, mitochondrial protective agents may be a good promising strategy form the inhibition of anticancer drugs toxicity. Mitochondrial permeability transition pore (MPTP) plays a central role in alterations of mitochondrial structure and function leading to neuronal injury [39]. Many anticancer drugs like studied ones in this work, trigger the formation of mPTP, resulting in increased oxidative stress, impaired mitochondrial respiration function, decreased mitochondrial membrane potential and release of cytochrome c [40]. Therefore, mitochondrial membrane permeability inhibitors can block the effects dependent on the opening of the MPT pore. Inhibition of MPTP has appeared as a promising approach for neuroprotection and development of well-tolerated mPT inhibitors with favorable blood-brain barrier penetration is highly warranted [41]. 28 clinically available drugs with a common heterocyclic structure were identified as mPT inhibitors [41]. In the current study we tested neuroprotective effect of TFP as a mPT inhibitor [42] against mitochondrial and oxidative stress induced by Ara-C, and IFOS. Our results showed that TFP as an antipsychotic drug and inhibitor of mPT reversed all the toxicities induced by Ara-C, and IFOS.

In summary our result confirmed that Ara-C, and IFOS induce cytotoxicity through mitochondrial dysfunction, oxidative stress and lysosomal damages in CNS neurons. Also, quite interestingly our data showed that TFP as an antipsychotic drug and inhibitor of $\mathrm{mPT}$ with good penetration through blood-brain barrier can reverse Ara-C, and IFOS -induced neurotoxicity in isolated neurons.

\section{Acknowledgement}

The data provided in this article was extracted from the Pharm D. theses of Dr. Shadi Headari Nik and Dr. Adineh Khodadoost. The theses were conducted under supervision of Prof. Jalal Pourahmad at Department of Toxicology and Pharmacology, Faculty of Pharmacy, Shahid Beheshti University of Medical Sciences, Tehran, Iran. This study was supported by Kermanshah University of Medical Sciences, Deputy of Research.

\section{Conflict of Interest}

The authors declare that they have no conflict of interest.

\section{References}

[1] Bhagra A, Rao RD. Chemotherapy-induced neuropathy. Current Oncology Reports 2007; 9: 290-299

[2] Park SB, Goldstein D, Krishnan AV et al. Chemotherapy-induced peripheral neurotoxicity: A critical analysis. CA: A Cancer Journal for Clinicians 2013; 63: 419-437 
[3] Areti A, Yerra VG, Naidu V et al. Oxidative stress and nerve damage: Role in chemotherapy induced peripheral neuropathy. Redox Biology 2014; 2: 289-295

[4] Tong L, Chuang C-C, Wu S et al. Reactive oxygen species in redox cancer therapy. Cancer Letters 2015; 367: 18-25

[5] Ye Z-W, Zhang J, Townsend DM et al. Oxidative stress, redox regulation and diseases of cellular differentiation. Biochimica et Biophysica Acta (BBA)-General Subjects 2015; 1850: 1607-1621

[6] Gogvadze V, Orrenius S, Zhivotovsky B. Mitochondria as targets for chemotherapy. Apoptosis 2009; 14: 624-640

[7] Bozza FA, D’Avila JC, Ritter C et al. Bioenergetics, mitochondrial dysfunction, and oxidative stress in the pathophysiology of septic encephalopathy. Shock 2013; 39: 10-16

[8] Figueira TR, Barros MH, Camargo AA et al. Mitochondria as a source of reactive oxygen and nitrogen species: From molecular mechanisms to human health. Antioxidants \& Redox Signaling 2013; 18: 2029-2074

[9] Shipley JL, Butera JN. Acute myelogenous leukemia. Experimental Hematology 2009; 37: 649-658

[10] Zhuo M, Gorgun MF, Englander EW. Neurotoxicity of cytarabine (Ara-C) in dorsal root ganglion neurons originates from impediment of mtDNA synthesis and compromise of mitochondrial function. Free Radical Biology and Medicine 2018; 121: 9-19

[11] Lee BS, Lee JH, Kang H-G et al. Ifosfamide nephrotoxicity in pediatric cancer patients. Pediatric Nephrology 2001; 16: 796-799

[12] Hamadani M, Awan F. Role of thiamine in managing ifosfamideinduced encephalopathy. Journal of Oncology Pharmacy Practice 2006; 12: 237-239

[13] Salimi A, Pirhadi R, Jamali Z et al. Mitochondrial and lysosomal protective agents ameliorate cytotoxicity and oxidative stress induced by cyclophosphamide and methotrexate in human blood lymphocytes. Human \& Experimental Toxicology 2019; 38: 1266-1274

[14] Lee CS, Park SY, Ko HH et al. Inhibition of MPP + -induced mitochondrial damage and cell death by trifluoperazine and W-7 in PC12 cells. Neurochemistry International 2005; 46: 169-178

[15] Han JH, Kim YJ, Han ES et al. Prevention of 7-ketocholesterol-induced mitochondrial damage and cell death by calmodulin inhibition. Brain Research 2007; 1137: 11-19

[16] Rodrigues T, Santos AC, Pigoso AA et al. Thioridazine interacts with the membrane of mitochondria acquiring antioxidant activity toward apoptosis-potentially implicated mechanisms. British Journal of Pharmacology 2002; 136: 136-142

[17] Liu S, Han Y, Zhang T et al. Protective effect of trifluoperazine on hydrogen peroxide-induced apoptosis in PC12 cells. Brain Research Bulletin 2011; 84: 183-188

[18] Pereira RS, Bertocchi APF, Vercesi AE. Protective effect of trifluoperazine on the mitochondrial damage induced by $\mathrm{Ca} 2+$ plus prooxidants. Biochemical Pharmacology 1992; 44: 1795-1801

[19] Yan L, Zeng Q, Wang J et al. Dexmedetomidine reduces propofolinduced apoptosis of neonatal rat hippocampal neurons via up-regulating Bcl-2 expression. Biomedical Research 2018; 29: 1199-1204

[20] Faizi M, Salimi A, Rasoulzadeh Met al. Schizophrenia induces oxidative stress and cytochrome $c$ release in isolated rat brain mitochondria. Iranian Journal of Pharmaceutical Research 2014; 13: 93-100

[21] Assadian E, Zarei MH, Gilani AG et al. Toxicity of Copper Oxide (CuO) Nanoparticles on Human Blood Lymphocytes. Biological Trace Element Research 2018: 184 350-357

[22] Boya P, Kroemer G. Lysosomal membrane permeabilization in cell death. Oncogene 2008; 27: 6434

[23] Beach DC, Giroux E. Inhibition of lipid peroxidation promoted by iron (III) and ascorbate. Archives of Biochemistry and Biophysics 1992; 297 : 258-264
[24] Hissin P], Hilf R. A fluorometric method for determination of oxidized and reduced glutathione in tissues. Analytical Biochemistry 1976; 74 : 214-226

[25] Lushchak VI. Free radicals, reactive oxygen species, oxidative stress and its classification. Chemico-Biological Interactions 2014; 224: 164-175

[26] Deavall DG, Martin EA, Horner JM et al. Drug-induced oxidative stress and toxicity. J Toxicol 2012; 2012: 645460. doi: 10.1155/2012/ 645460

[27] Sharma P, Jha AB, Dubey RS, Pessarakli M Reactive oxygen species, oxidative damage, and antioxidative defense mechanism in plants under stressful conditions. Journal of Botany 2012; 2012: doi: $10.1155 / 2012 / 217037$

[28] Froklage FE, Reijneveld JC, Heimans J]. Central neurotoxicity in cancer chemotherapy: pharmacogenetic insights. Pharmacogenomics 2011; 12: 379-395

[29] Yokoyama C, Sueyoshi Y, Ema M et al. Induction of oxidative stress by anticancer drugs in the presence and absence of cells. Oncology Letters 2017; 14: 6066-6070

[30] Zhang J, Lei W, Chen X et al. Oxidative stress response induced by chemotherapy in leukemia treatment. Molecular and Clinical Oncology 2018; 8: 391-399

[31] Chan K, Truong D, Shangari N et al. Drug-induced mitochondrial toxicity. Expert Opinion on Drug Metabolism \& Toxicology 2005; 1: 655-669

[32] Schapira AH. Mitochondrial disease. The Lancet 2006; 368: 70-82

[33] Will Y, Shields JE, Wallace KB. Drug-induced mitochondrial toxicity in the geriatric population: Challenges and future directions. Biology 2019; 8: 32

[34] Aits S, Jäättelä M Lysosomal Cell Death at a Glance. The Company of Biologists Ltd: 2013

[35] Woldemichael T, Rosania GR. The physiological determinants of drug-induced lysosomal stress resistance. PLoS One 2017; 12: e0187627

[36] Moustapha A, Pérétout P, Rainey $\mathrm{N}$ et al. Curcumin induces crosstalk between autophagy and apoptosis mediated by calcium release from the endoplasmic reticulum, lysosomal destabilization and mitochondrial events. Cell Death Discovery 2015; 1: 15017

[37] Mut-Salud N, Álvarez PJ, Garrido JM et al. Antioxidant intake and antitumor therapy: Toward nutritional recommendations for optimal results. Oxid Med Cell Longev 2016; 2016: 6719534. doi: $10.1155 / 2016 / 6719534$

[38] Guo C, Sun L, Chen X et al. Oxidative stress, mitochondrial damage and neurodegenerative diseases. Neural Regeneration Research 2013; 8: 2003

[39] Shevtsova FE, Vinogradova VD, Neganova EM et al. Mitochondrial permeability transition pore as a suitable target for neuroprotective agents against Alzheimer's disease. CNS \& Neurological DisordersDrug Targets (Formerly Current Drug Targets-CNS \& Neurological Disorders) 2017; 16: 677-685

[40] Gorini S, De Angelis A, Berrino L, et al. Chemotherapeutic drugs and mitochondrial dysfunction: Focus on doxorubicin, trastuzumab, and sunitinib. Oxidative Medicine and Cellular Longevity 2018, 2018

[41] Morota S, Månsson R, Hansson MJ et al. Evaluation of putative inhibitors of mitochondrial permeability transition for brain disordersSpecificity vs. toxicity. Experimental Neurology 2009; 218: 353-362

[42] Banerjee S, Melnyk SB, Krager KJ et al. Trifluoperazine inhibits acetaminophen-induced hepatotoxicity and hepatic reactive nitrogen formation in mice and in freshly isolated hepatocytes. Toxicology Reports 2017; 4: 134-142 